\title{
MicroRNA-29a enhances cisplatin sensitivity in non-small cell lung cancer through the regulation of REV3L
}

\author{
XIALIN CHEN $^{1 *}$, HONG ZHU $^{2 *}$, WANLI YE ${ }^{1}$, YAYUN CUI $^{3}$ and MING CHEN ${ }^{4}$ \\ ${ }^{1}$ Department of Oncology, The Second Affiliated Hospital of Soochow University, Gusu, Suzhou, Jiangsu 215000; \\ ${ }^{2}$ Department of Radiation Oncology, Minhang Branch of Cancer Hospital of Fudan University, Shanghai 200240; \\ ${ }^{3}$ Department of Radiation Oncology, The Affiliated Provincial Hospital of Anhui Medical University, Hefei, Anhui 230000; \\ ${ }^{4}$ Department of Radiation Oncology, Zhejiang Cancer Hospital, Gongshu, Hangzhou, Zhejiang 310000, P.R. China
}

Received April 5, 2018; Accepted September 28, 2018

DOI: $10.3892 / \mathrm{mmr} .2018 .9723$

\begin{abstract}
Cisplatin-based chemotherapy may greatly enhance patient prognosis; however, chemotherapy resistance remains an obstacle to curing patients with non-small cell lung cancer (NSCLC). The aim of the present study was to explore the microRNAs (miRs) that could regulate cisplatin sensitivity and provide a potential treatment method for cisplatin resistance in clinical. Results from the present study revealed that miR-29a overexpression enhanced and miR-29a inhibition reduced the sensitivity of two NSCLC cell lines, A549 and $\mathrm{H} 1650$, to cisplatin treatment. In addition, reduced miR-29a expression levels were observed in cisplatin-resistant A549 cells (A549rCDDP), and increased expression of miR-29a augmented cisplatin-induced inhibition of proliferation and apoptosis in A549rCDDP cells. These data indicated that miR-29a expression may be involved in the development of cisplatin resistance. miR-29a was revealed to negatively regulate REV3-like DNA-directed polymerase $\zeta$ catalytic subunit (REV3L) expression in both A549 and H1650 cells; elevated expression of REV3L in A549rCDDP cells was also detected. REV3L encodes the catalytic subunit of DNA polymerase $\zeta$ and was hypothesized, based on results from the online tool TargetScan 7.1, to be a target gene of miR-29a; this was confirmed with a dual luciferase assay. Cells treated with a very low concentration of cisplatin exhibited a significant reduction
\end{abstract}

Correspondence to: Dr. Yayun Cui, Department of Radiation Oncology, The Affiliated Provincial Hospital of Anhui Medical University, 17 Lujiang Road, Hefei, Anhui 230000, P.R. China

E-mail: 83305848@qq.com

Dr Ming Chen, Department of Radiation Oncology, Zhejiang Cancer Hospital, 1 Banshan Road, Gongshu, Hangzhou, Zhejiang 310000, P.R. China

E-mail: chenming@zjcc.org.cn

*Contributed equally

Key words: microRNA-29a, cisplatin sensitivity, non-small-cell lung cancer, REV3-like DNA-directed polymerase $\zeta$ catalytic subunit in proliferation and cell cycle arrest at the $\mathrm{G} 2 / \mathrm{M}$ phase in REV3L-knockdown as well as in miR-29a-upregulated A549 cells. Notably, reduced miR-29a expression and an increase in REV3L mRNA expression were observed in tumor tissues from patients with NSCLC. Additionally, a negative correlation between miR-29a and REV3L mRNA expression levels in tumor tissues from patients with NSCLC was observed; low expression of miR-29a and high expression of REV3L were closely associated with an advanced tumor-node-metastasis classification. The results of the present study suggested a pivotal role of miR-29a in mediating NSCLC cell sensitivity towards cisplatin through the regulation of REV3L.

\section{Introduction}

Lung cancer remains a leading cause of cancer-related mortality worldwide (1). Non-small cell lung cancer (NSCLC) is a major type of lung cancer and is characterized by a poor prognosis with relatively low 5-year survival rate $(2,3)$. Cisplatin-based chemotherapy is a standard procedure for the treatment of patients with NSCLC (4). Many patients initially respond to cisplatin-based chemotherapy, whereas certain patients with intrinsic resistance do not initially respond to cisplatin and others develop acquired resistance to cisplatin (5). As a result, the 5-year survival rate for patients with NSCLC is only $17 \%$ (6).

MicroRNAs (miRNAs) are non-coding, short single-chain nucleotide molecules (7). Through binding to the 3'-untranslated region (UTR) of their target genes, miRNAs control a variety of physiological processes, including cell proliferation, cell migration and the cell cycle (8). Dysregulation of miRNAs has been reported to contribute to the development of multiple diseases such as cancer $(9,10)$. In addition, a number of miRNAs have been reported to be involved in the development of chemoresistance $(11,12)$. miRNA (miR)-29 has been demonstrated to sensitize ovarian cancer cells to cisplatin treatment, and represents a potential therapeutic target (13). However, whether and how miR-29 contributes to the development of cisplatin resistance in NSCLC remains unknown.

REV3-like DNA-directed polymerase $\zeta$ catalytic subunit (REV3L) encodes the catalytic subunit of DNA polymerase $\zeta$, which is responsible for translesional replication (14); this 
function makes REV3L a cancer susceptibility candidate gene. A previous study reported that a c.460T $>C$ variant in the REV3L 3'UTR affected the binding ability of miRNAs on REV3L mRNA and may contribute to lung cancer initiation (15). Despite its role in cancer initiation, overexpression of REV3L has been reported to promote cell survival and the development of cisplatin resistance in human fibroblasts (16).

In the present study, the expression of miR-29a was demonstrated to determine the sensitivity of A549 and H1650 cells to cisplatin. Furthermore, miR-29a expression was reduced in the cisplatin resistant A549 cell line (A549rCDDP), and increased miR-29a expression resensitized A549rCDDP cells to cisplatin. REV3L was confirmed to be a target gene of miR-29a. Further investigations revealed that the silencing of REV3L expression and the overexpression of miR-29a in A549 cells that were treated with a low concentration of cisplatin may significantly reduce cell proliferation, inhibition and cell cycle arrest at the G2/M phase. In addition, a decrease in miR-29a expression and an increase in REV3L expression were observed in cisplatin-resistant A549rCDDP cells. Gene expression analysis in tumor tissues from patients with NSCLC revealed a negative correlation between miR-29a and REV3L mRNA expression. In conclusion, results from the present study indicated that miR-29a may enhance NSCLC cell sensitivity to cisplatin treatment through the regulation of REV3L expression.

\section{Materials and methods}

Cell culture. The human NSCLC cell lines A549 and H1650, and 293 cells were purchased from American Type Culture Collection (Manassas, VA, USA), The A549 cisplatin-resistant sub-line, A549rCDDP, was obtained from The Cancer Hospital of Peking Union Medical College, Chinese Academy of Medical Sciences (Beijing, China). All cell lines were cultured in RPMI-1640 medium (Gibco; Thermo Fisher Scientific, Waltham, MA, USA) supplemented with $10 \%$ fetal bovine serum (HyClone; GE Healthcare Life Sciences, Logan, UT, USA) in a humid incubator with $5 \% \mathrm{CO}_{2}$. For A549rCDDP cells, the complete culture medium was supplemented with $2 \mathrm{mg} / \mathrm{l}$ cisplatin (Selleck Chemicals, Houston, TX, USA). For cisplatin treatment conditions, the culture medium of A549, H1650 or A549rCDDP cells was supplemented with cisplatin $(2.5,5,10$ and $20 \mu \mathrm{g} / \mathrm{ml}$; Sigma-Aldrich; Merck KGaA, Darmstadt, Germany) for the indicated times (24, 48 and $72 \mathrm{~h})$.

Small interfering (si)RNA transfection. Two REV3L siRNAs (\#1, 5'-GAUCACAGGUUUGUGCCAG-3'; and \#2, 5'-AGA CUGAGUGAGUCACCUG-3') and a control siRNA were purchased from Invitrogen (Thermo Fisher Scientific, Inc.). Cells $\left(2 \times 10^{5}\right)$ were seeded in 6-well plates and cultured for $24 \mathrm{~h}$; the siRNAs were individually mixed with Lipofectamine ${ }^{\circledR}$ RNAiMAX (Invitrogen; Thermo Fisher Scientific, Inc.) and added into the cell culture medium at a final concentration of $0.01 \mu \mathrm{M}$ and incubated for $72 \mathrm{~h}$ according to the manufacturer's instructions. At $72 \mathrm{~h}$ after transfection, cells were collected for the subsequent experiments.
miRNA transfection. Cells were transfected with $50 \mathrm{nmol} / \mathrm{l}$ miR-29a mimics (5'-UAGCACCAUCUGAAAUCGGUUA-3') or miR-NC mimics (5'-UAACCACUUUCACAUGGUCCUA3'), miR-29a inhibitor (5'-UAACCGAUUUCAGAUGGU GCUA-3') or miR-NC inhibitor (5'-UAACCGAAUUCACAU GGUCCUA-3') using Lipofectamine ${ }^{\circledR} 2000$ (Thermo Fisher Scientific, Inc.). In brief, cells $\left(2 \times 10^{5}\right)$ were seeded in a 6 -well plate and incubated to $60-70 \%$ confluence. At $24 \mathrm{~h}$ after transfection, cells were collected for the subsequent experiments.

Cell cycle assay. For cell cycle analysis, cells were stained with propidium iodide (PI; Invitrogen; Thermo Fisher Scientific, Inc.). Briefly, following different treatments (control siRNA + miR-NC mimics + vehicle; control siRNA + miR-NC mimics + cisplatin; REV3L siRNA1 + miR-NC mimics + cisplatin; REV3L siRNA2 + miR-NC mimics + cisplatin; and control siRNA + miR-29a mimics + cisplatin) in five groups, cells were collected, washed with PBS and fixed in $70 \%$ ethanol at $4^{\circ} \mathrm{C}$ overnight. Annexin V $(5 \mu \mathrm{l})$ and PI $(2.5 \mu \mathrm{l})$ were subsequently added to the cell suspension, and cell distribution was analyzed by flow cytometry. The cell number at each phase was analyzed using FloJo software (version 7.6.3; FlowJo LLC, Ashland, OR, USA).

RNA extraction and reverse transcription-quantitative polymerase chain reaction $(R T-q P C R)$. MiRNeasy Mini kit (Qiagen, Inc. Valencia, CA, USA) was used to extract total RNA from cells, according to the manufacturer's instructions. An M-MLV Reverse Transcriptase kit (Thermo Fisher Scientific, Inc.) was used to synthesize cDNA. qPCR was performed using SYBR Premix Ex Taq (Takara Bio, Inc., Otsu, Japan) on a CFX96 Real-Time PCR Detection System (Bio-Rad Laboratories, Hercules, CA, USA). GAPDH and U6 were used as internal controls for mRNA and miRNA, respectively. The primers were as follows: miR-29a, 5'-TAG CACCATCTGAAATCG-3' (forward) and 5'-CACACCAGC ACTGACTA-3' (reverse); GAPDH, 5'-TGAACTGAAAGC TCTCCACC-3' (forward) and 5'-CTGATGTACCAGTTG GGGAA-3' (reverse); U6, 5'-CTCGCTTCGGCAGCACA-3' (forward), 5'-AACGCTTCACGAATTTGCGT-3' (reverse); REV3L, 5'-GCTCCAGTATGT GTACCATCT TGT-3' (forward) and 5'-ATGGATATCTCGAAGTAACACGTC-3' (reverse). The $2^{-\triangle \Delta C q}$ method was used to calculate relative gene expression (17).

Western blotting. Cell lysates $\left(100 \mu \mathrm{l} ; 2 \times 10^{6}\right.$ cells) were prepared using radioimmunoprecipitation assay lysis buffer (Beyotime Institute of Biotechnology, Haimen, China) containing $2 \mu \mathrm{l}$ protease inhibitor (Sigma-Aldrich; Merck $\mathrm{KGaA}$ ). Briefly, the concentration of each protein sample was determined by bicinchoninic acid assay kit (Beyotime Institute of Biotechnology), and the total protein $(20 \mu \mathrm{g} / \mathrm{lane})$ extracted from each sample was separated by SDS-PAGE on $8 \%$ gels and transferred to polyvinylidene fluoride membranes (EMD Millipore, Billerica, MA, USA). The membranes were blocked in 5\% non-fat milk and incubated with primary antibodies against REV3L (1:1,000; catalog no. GTX17515; GeneTex, Inc., Irvine, CA, USA) and GAPDH (1:10,000; catalog no. G8795; Sigma-Aldrich; Merck KGaA) at $4^{\circ} \mathrm{C}$ overnight, followed by incubation with anti-rabbit peroxidase-conjugated secondary 
Table I. Association between miR-29a or REV3L and clinicopathological factors.

\begin{tabular}{|c|c|c|c|c|c|}
\hline \multirow{2}{*}{$\begin{array}{l}\text { Clinicopathological } \\
\text { parameters }\end{array}$} & \multirow[b]{2}{*}{$\mathrm{n}$} & \multicolumn{2}{|c|}{ miR-29a expression } & \multicolumn{2}{|c|}{ REV3L expression } \\
\hline & & Mean \pm SD & P-value & Mean \pm SD & P-value \\
\hline Age & & & 0.686 & & 0.435 \\
\hline$<60$ & 9 & $0.749 \pm 0.094$ & & $1.422 \pm 0.141$ & \\
\hline$\geq 60$ & 21 & $0.707 \pm 0.054$ & & $1.305 \pm 0.076$ & \\
\hline Sex & & & 0.817 & & 0.927 \\
\hline Male & 20 & $0.735 \pm 0.101$ & & $1.349 \pm 0.122$ & \\
\hline Female & 10 & $0.712 \pm 0.051$ & & $1.336 \pm 0.083$ & \\
\hline TNM classification & & & $0.007^{\mathrm{a}}$ & & $0.003^{\mathrm{a}}$ \\
\hline I-II & 13 & $0.867 \pm 0.061$ & & $1.108 \pm 0.059$ & \\
\hline III-IV & 17 & $0.621 \pm 0.056$ & & $1.495 \pm 0.089$ & \\
\hline Metastasis & & & 0.264 & & 0.059 \\
\hline Yes & 18 & $0.676 \pm 0.063$ & & $1.443 \pm 0.085$ & \\
\hline No & 12 & $0.784 \pm 0.067$ & & $1.185 \pm 0.099$ & \\
\hline
\end{tabular}

aP $<0.01$. miR, microRNA; REV3L, REV3-like DNA-directed polymerase $\zeta$ catalytic subunit; SD, standard deviation; TNM, tumor-node-metastasis .
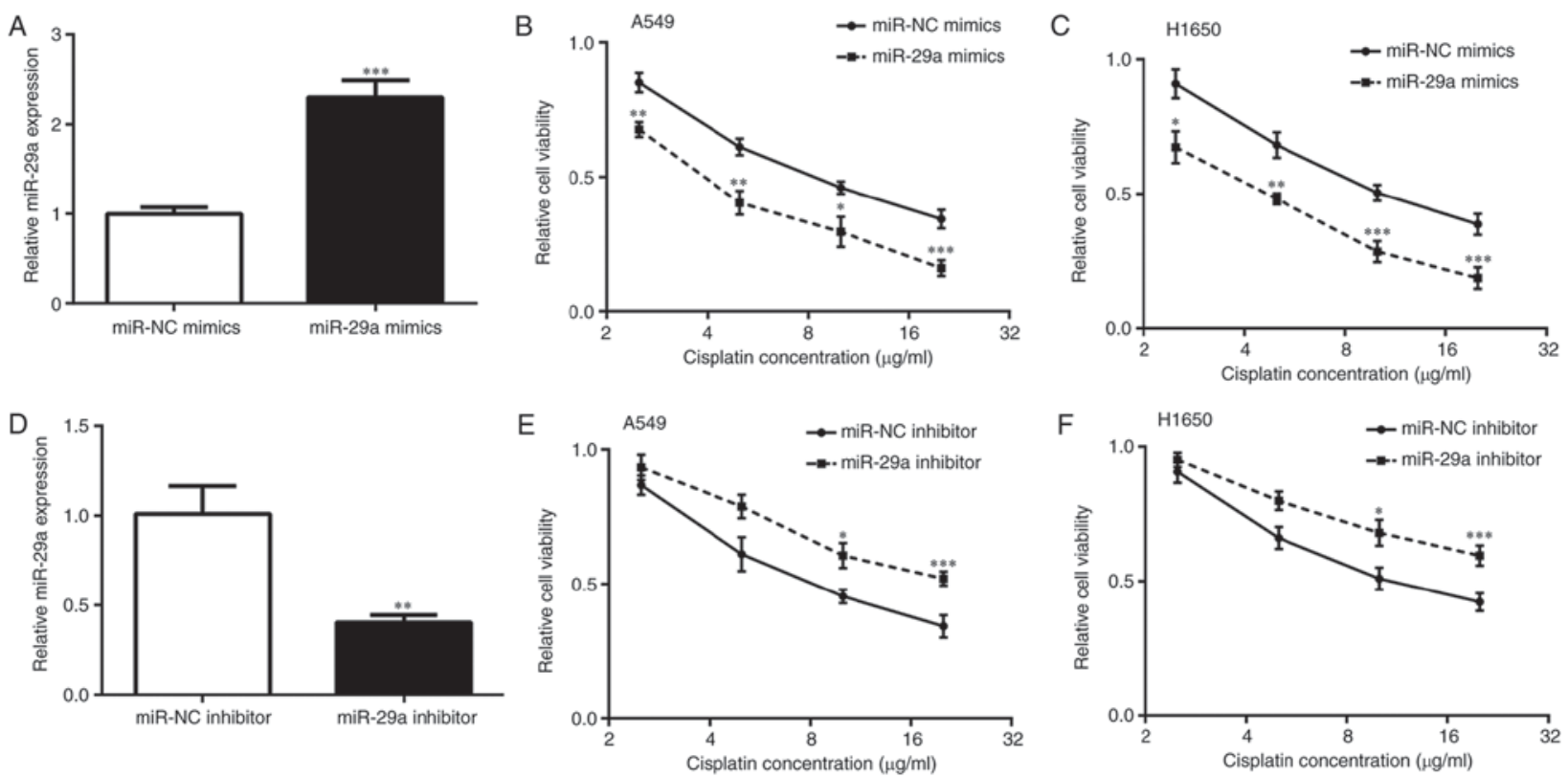

Figure 1. miR-29a regulates cisplatin sensitivity of non-small cell lung cancer cell lines A549 and H1650. (A) Transfection of miR-29a mimics increased miR-29a expression levels in A549 cells, as determined by RT-qPCR. (B and C) Overexpression of miR-29a enhanced the sensitivity of (B) A549 and (C) H650 cells towards cisplatin treatment, as determined by the reduced viability of transfected cells. (D) Transfection of miR-29a inhibitor decreased miR-29a expression levels in A549 cells, as determined by RT-qPCR. Downregulation of miR-29a desensitized (E) A549 and (F) H1650 cells towards cisplatin treatment, as determined by increased viability in treated sells. ${ }^{*} \mathrm{P}<0.05,{ }^{* * *} \mathrm{P}<0.01$ and ${ }^{* * * *} \mathrm{P}<0.0001$ vs. miR-NC. miR, microRNA; NC, negative control; RT-qPCR, reverse transcription-quantitative polymerase chain reaction.

antibody (1:80,000; catalog no. a0545; Sigma-Aldrich; Merck $\mathrm{KGaA}$ ) at room temperature for $1 \mathrm{~h}$. Protein bands were visualized using Enhanced Chemiluminescence detection reagents (Thermo Fisher Scientific, Inc. USA). GAPDH served as a loading control.

Cell viability assay. Cell viability was determined by Cell Counting Kit-8 (Dojindo Molecular Technologies, Inc., Kumamoto, Japan). For the detection of miR-29a on cisplatin induced cell viability, cells were seeded in a 96-well plate and subsequently exposed to vehicle $(0.9 \% \mathrm{NaCl}$ as control for ciaplatin) or cisplatin treatments $(2.5,5,10$ and $20 \mu \mathrm{g} / \mathrm{ml})$ for $72 \mathrm{~h}$.

For the determining the effect of miR-29a on cisplatin induced changes of cell proliferation, cells were treated with cisplatin $(5 \mu \mathrm{g} / \mathrm{ml})$ for $72 \mathrm{~h}$. Subsequently, cells $\left(2 \times 10^{5}\right)$ were seeded in a 6 -well plate and transfected with $50 \mathrm{nmol} / 1 \mathrm{miR}-29 \mathrm{a}$ mimics, miR-29a inhibitor or NC using Lipofectamine ${ }^{\circledR} 2000$ 

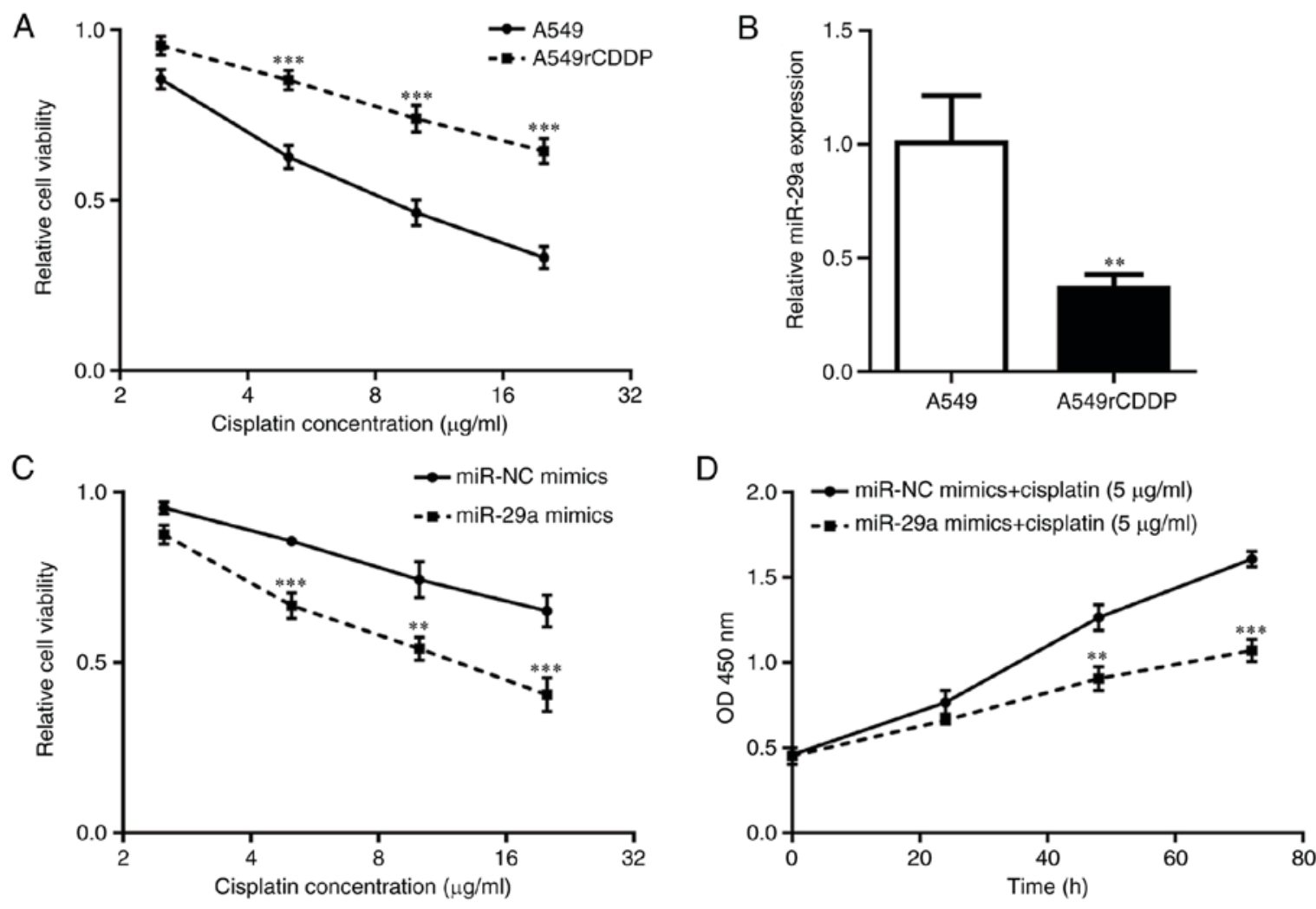

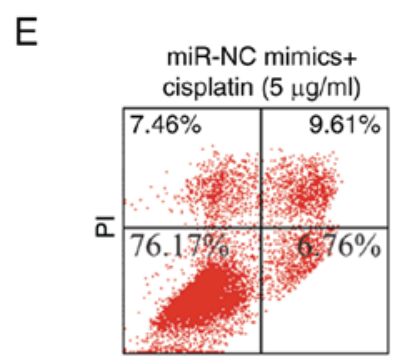

Annexin

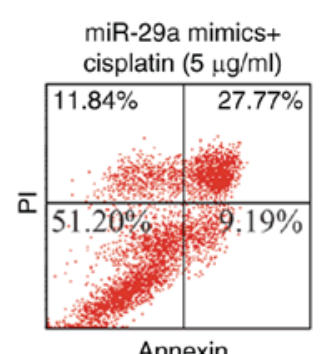

Annexin

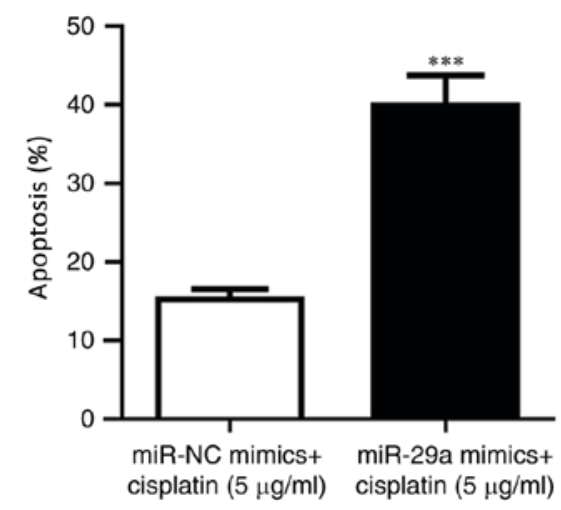

Figure 2. Upregulation of REV3L contributed to cisplatin resistance of A549rCDDP cells. (A) Compared with A549 cells, cisplatin-resistant A549rCDDP cells were relatively insensitive towards cisplatin treatment, as indicated by the significantly higher cell viability; ${ }^{* * *} \mathrm{P}<0.001$ vs. A549. (B) miR-29a expression levels were significantly lower in A549rCDDP cells compared with A549 cells, as determined by reverse transcription-quantitative polymerase chain reaction (C) Overexpression of miR-29a increased sensitivity of A549rCDDP cells towards cisplatin treatment, as indicated by the reduction in viability; ${ }^{* *} \mathrm{P}<0.01$ and ${ }^{* * * *} \mathrm{P}<0.001$ vs. miR-NC. (D) miR-29a overexpression reduced cell proliferation in the presence of cisplatin in A549rCDDP cells; ${ }^{* *} \mathrm{P}<0.01$ and ${ }^{* * * *} \mathrm{P}<0.001$ vs miR-NC + cisplatin. (E) miR-29a overexpression significantly increased cell apoptosis rate in the presence of cisplatin in A549rCDDP cells. miR, microRNA; NC, negative control; OD, optical density; PI, propidium iodide; REV3L, REV3-like DNA-directed polymerase $\zeta$ catalytic subunit.

(Thermo Fisher Scientific, Inc.). Subsequently, at $24 \mathrm{~h}$ after transfection, cells were collected for the subsequent experiments.

To determine the effect of REV3L on cell viability, REV3L siRNA $(0.01 \mu \mathrm{M})$ or control siRNA (Thermo Fisher Scientific, Inc.) was transfected into cells which were treated with cisplatin $(2 \mu \mathrm{g} / \mathrm{ml})$ by Lipofectamine ${ }^{\circledR}$ RNAiMAX (Invitrogen; Thermo Fisher Scientific, Inc.). At $72 \mathrm{~h}$ after transfection, cells were collected for the subsequent experiments. Briefly, $10 \mu$ l CCK- 8 was added to the culture medium of each well and incubated for $2 \mathrm{~h}$. The absorbance was measured at $450 \mathrm{~nm}$ with a microplate reader (Bio-Rad Laboratories, Inc.).
Subsequently, $10 \mu 1$ CCK- 8 was added to the culture medium of each well and incubated for $2 \mathrm{~h}$. The absorbance was measured at $450 \mathrm{~nm}$ with a microplate reader (Bio-Rad Laboratories).

Cell apoptosis assay. Cells were collected by trypsinization and cell apoptosis was detected using an Annexin V-fluorescein isothiocyanate/PI cell apoptosis kit (Invitrogen; Thermo Fisher Scientific, Inc.), according to the manufacturer's instructions. Briefly, cells were suspended in Annexin binding buffer, and PI and Annexin V were added to the cell suspension. Cells were analyzed with a BD FACSCalibur flow cytometer (BD Biosciences, Franklin Lakes, NJ, USA). 



B

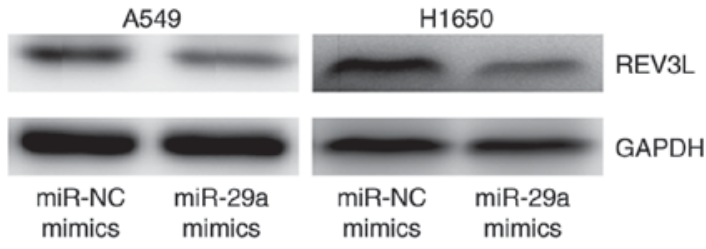

D

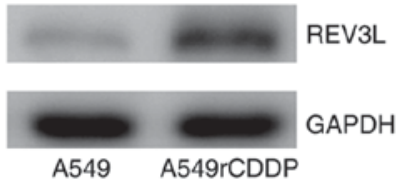

$\mathrm{F}$

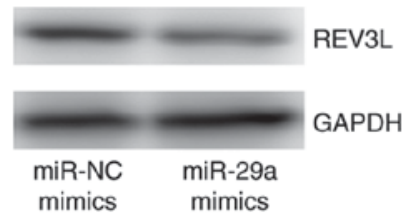

Figure 3. REV3L is negatively regulated by miR-29a in non-small cell lung cancer cell lines cells. (A and B) Overexpression of miR-29a decreased REV3L (A) mRNA and (B) protein expression levels in A549 and H1650 cells, as detected by RT-qPCR and western blotting, respectively. (C) mRNA and (D) protein expression levels of REV3L were elevated in A549rCDDP cells in comparison with A549 cells. (E and F) Overexpression of miR-29a decreased REV3L (E) mRNA and $(\mathrm{F})$ protein expression levels in A549rCDDP cells. ${ }^{* * *} \mathrm{P}<0.001$. miR, microRNA; NC, negative control; REV3L, REV3-like DNA-directed polymerase $\zeta$ catalytic subunit; RT-qPCR, reverse transcription-quantitative polymerase chain reaction.

Luciferase reporter assay. The wild-type (WT) REV3L 3'UTR sequence was amplified from cDNA of 293 cells and inserted into pGL-3 (Promega Corporation, Madison, WI, USA). REV3L 3'UTR-mutant (Mut) was constructed using PrimeSTAR Mutagenesis Basal kit (Takara Bio, Inc.). The 293 cells were co-transfected with pGL3-REV3L 3'UTR-WT or pGL3-REV3L 3'UTR-Mut and miR-29a mimics or miR-negative control (NC) mimics, and an internal control Renilla plasmid. Luciferase activity and Renilla activity were measured at $24 \mathrm{~h}$ post-transfection using a Dual Luciferase Reporter Assay kit (Promega Corporation), according to the manufacturer's instructions.

Patients. NSCLC tissues and adjacent normal liver tissues (located $\geq 2 \mathrm{~cm}$ from the tumor margins) were obtained from 30 patients ( 20 male and 10 female; 9 patients aged $<60$ years old and 21 patients aged $\geq 60$ years old) who received surgery at Zhejiang Cancer Hospital (Zhejiang, China) and Shaoxing People's Hospital (Shaoxing, China; Table I). Patients that

\section{A REV3L 3'UTR 5'-CUAAACUGUUGUGCAUGGUGCUU-3' |||||| miR-29a 3'-AUUGGCUAAAGUCUACCACGAU-5'}

B

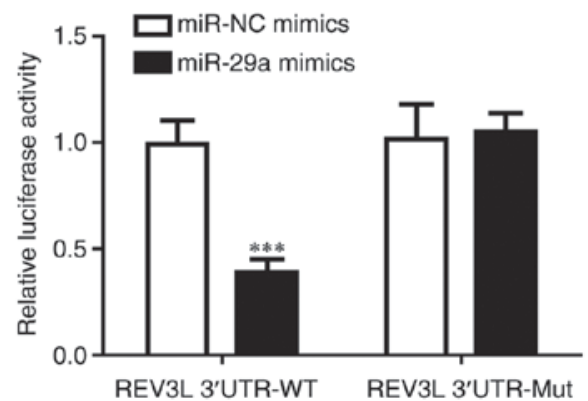

Figure 4. REV3L is a direct target of miR-29a. (A) Sequence alignment of REV3L 3'UTR and miR-29a. (B) 293 cells were co-transfected with miR-29a mimics or miR-NC mimics and REV3L 3'UTR-WT or REV3L 3'UTR-Mut. Transfection of miR-29a mimics significantly reduced luciferase activity in cells transfected with REV3L 3'UTR-WT. ${ }^{* * *} \mathrm{P}<0.001$. miR, microRNA; Mut, mutant; NC, negative control; REV3L, REV3-like DNA-directed polymerase $\zeta$ catalytic subunit; UTR, untranslated region; WT, wild-type. 
A

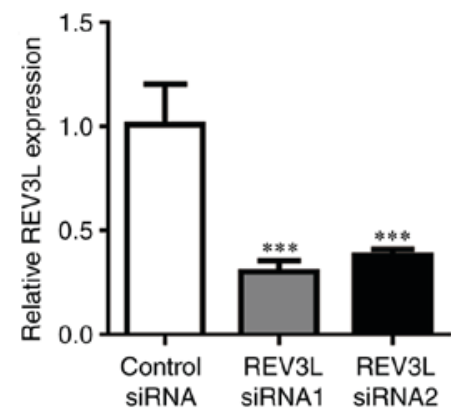

B



C
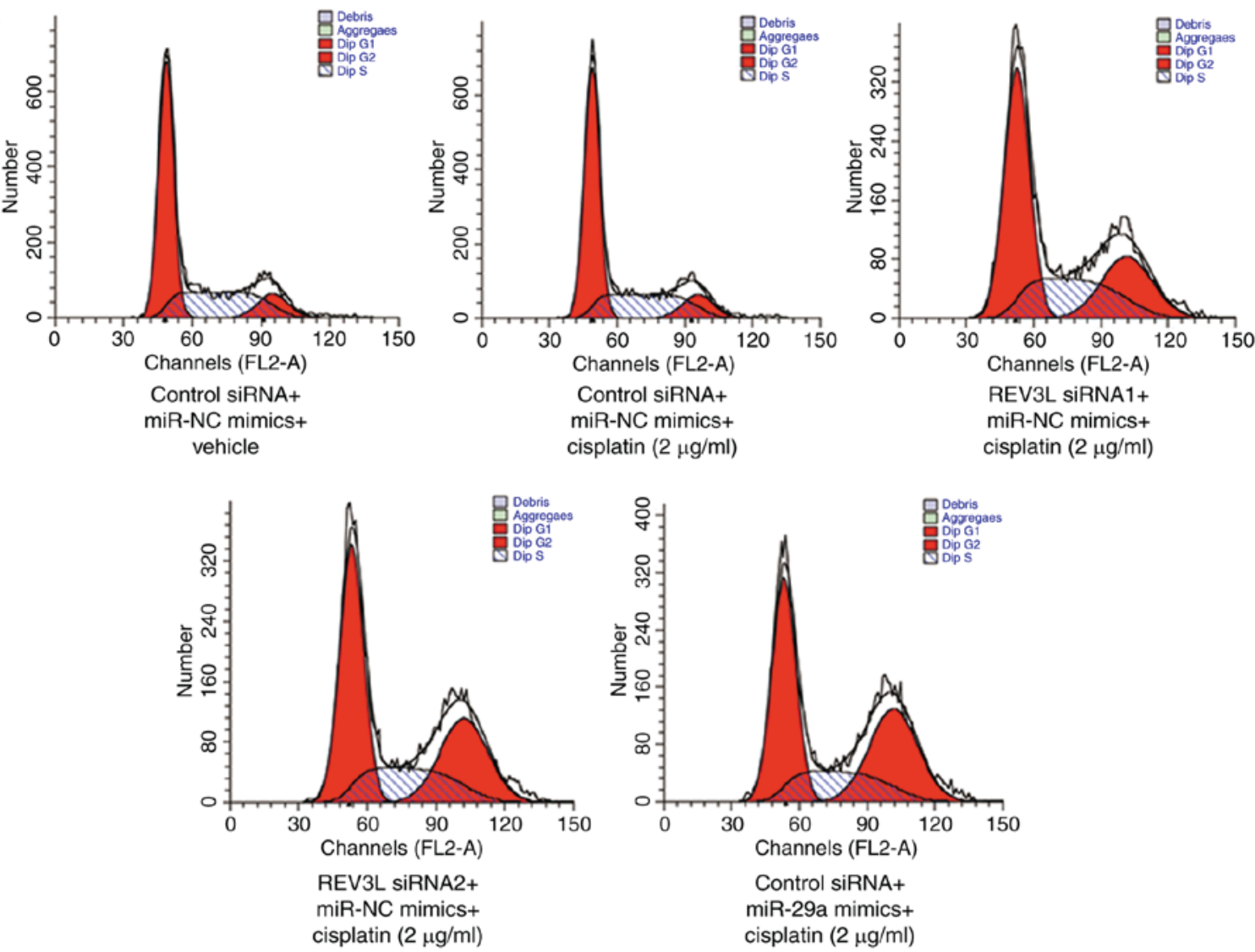

D

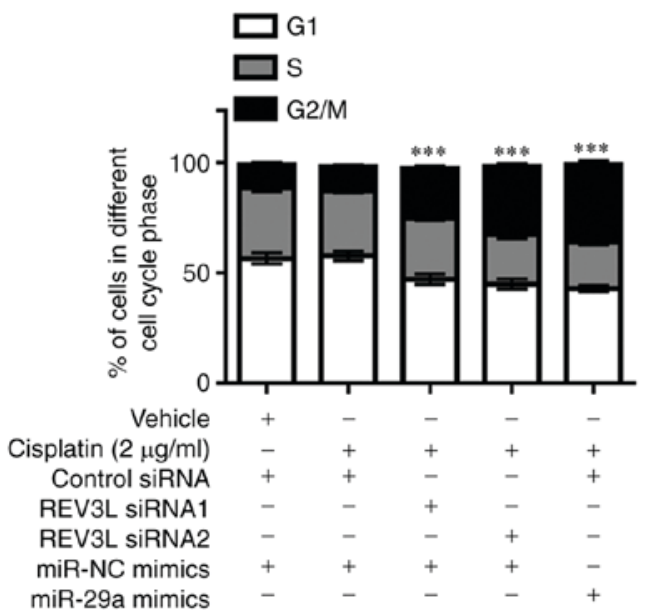

E

$\rightarrow$ Control siRNA+miR-NC mimics+vehicle

$\rightarrow$ - Control siRNA+miR-NC mimics+cisplatin $(2 \mu \mathrm{g} / \mathrm{ml})$

-1. REV3L siRNA1+miR-NC mimics+cisplatin $(2 \mu \mathrm{g} / \mathrm{ml})$

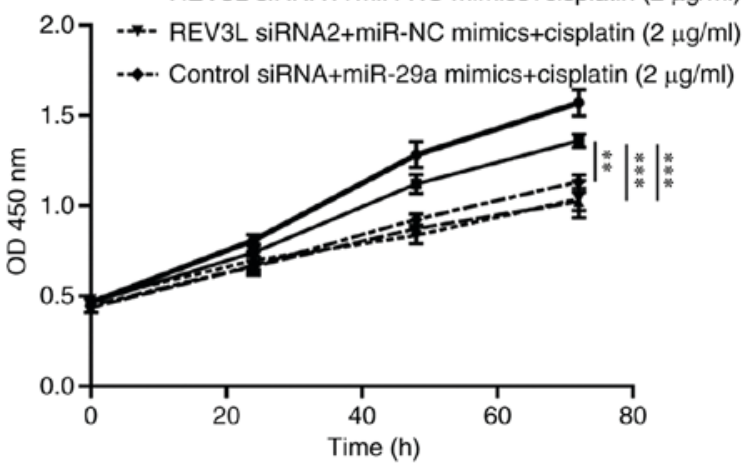

Figure 5. REV3L is involved in miR-29a-regulated cisplatin sensitivity through regulation of the cell cycle. (A) mRNA and (B) protein expression levels of REV3L were decreased following transfection of REV3L siRNA1 or siRNA2 in A549 cells, as detected by reverse transcription-quantitative polymerase chain reaction and western blotting, respectively. (C) Silencing of REV3L or overexpression of miR-29a induced G2/M arrest in A549 cells co-treated with low concentration of cisplatin $(2 \mu \mathrm{g} / \mathrm{ml})$. (D) Quantitative analysis of cell cycle distribution from (C). (E) Proliferation was inhibited in A549 cells transfected with REV3L siRNAs or miR-29a mimics and co-treated with low concentration of cisplatin $(2 \mu \mathrm{g} / \mathrm{ml}) .{ }^{* *} \mathrm{P}<0.01$ and ${ }^{* * *} \mathrm{P}<0.001 \mathrm{vs}$. Control siRNA + miR-NC mimics + cisplatin $(2 \mu \mathrm{g} / \mathrm{ml})$. miR, microRNA; NC, negative control; REV3L, REV3-like DNA-directed polymerase $\zeta$ catalytic subunit; siRNA, small interfering RNA. 
received chemotherapy or radiotherapy were excluded from the study. Tissues were removed and stored at $-80^{\circ} \mathrm{C}$. The present study was approved by the ethics committee of Zhejiang Cancer Hospital, and written informed consent was obtained from each patient prior to surgery and enrolment in the study.

Prediction of the target of miR-29a. TargetScan 7.1 was used to predict target sequences of miR-29a in the 3'UTR of REV3L (www.targetscan.org/vert_71/).

Statistical analysis. All statistical analyses were carried out using GraphPad Prism 5.0 software (GraphPad Software, Inc., La Jolla, CA, USA). Data are presented as the mean \pm standard deviation. Statistically significant differences between two groups were analyzed using Student's t-test. Differences between multiple groups were analyzed with one-way ANOVA, followed by a Newman-Keuls post-hoc test. Correlations were made using Pearson's correlation coefficient. $\mathrm{P}<0.05$ was considered to indicate a statistically significant difference.

\section{Results}

miR-29a expression is associated with cisplatin sensitivity in NSCLC cells. To explore whether miR-29a expression affected the sensitivity of NSCLC cells to cisplatin, miR-29a mimics were used to increase miR-29a expression in A549 cells (Fig. 1A). miR-29a overexpression reduced the viability and increased cisplatin sensitivity of cisplatin-sensitive A549 and H1650 cells, compared with the cells transfected with miR-NC mimics (Fig. 1B and $\mathrm{C}$, respectively). Conversely, cells transfected an miR-29a inhibitor exhibited decreased miR-29a expression levels, increased viability and a reduction in sensitivity of A549 and $\mathrm{H} 1650$ cells to cisplatin exposure, compared with miR-NC-transfected cells (Fig. 1D-F). These data suggested that miR-29a expression may be involved in cisplatin sensitivity of NSCLC cells.

Reduced miR-29a expression is associated with development of cisplatin resistance of $A 549$ cells. The contribution of miR-29a dysregulation to the development of cisplatin resistance in NSCLC cells was investigated. Compared with parental A549 cells, treatment of cisplatin only slightly reduced cell viability of cisplatin-resistant A549rCDDP cells (Fig. 2A), which indicated a relative insensitivity of these cells to cisplatin. RT-qPCR confirmed a significantly decreased expression level of miR-29a in A549rCDDP cells compared with A549 cells (Fig. 2B). Transfection of miR-29a mimics enhanced the reduction in viability induced by cisplatin treatment in A549rCDDP cells, compared with miR-NC transfected cells (Fig. 2C). It was also revealed that miR-29a overexpression significantly reduced cell proliferation in A549rCDDP cells treated with $5 \mu \mathrm{g} / \mathrm{ml}$ cisplatin, compared with miR-NC-transfected cell (Fig. 2D). In addition, miR-29a overexpression significantly increased cisplatin-induced apoptosis (Fig. 2E). These data suggested that reduced miR-29a expression may be involved in the development of cisplatin resistance in NSCLC cells.
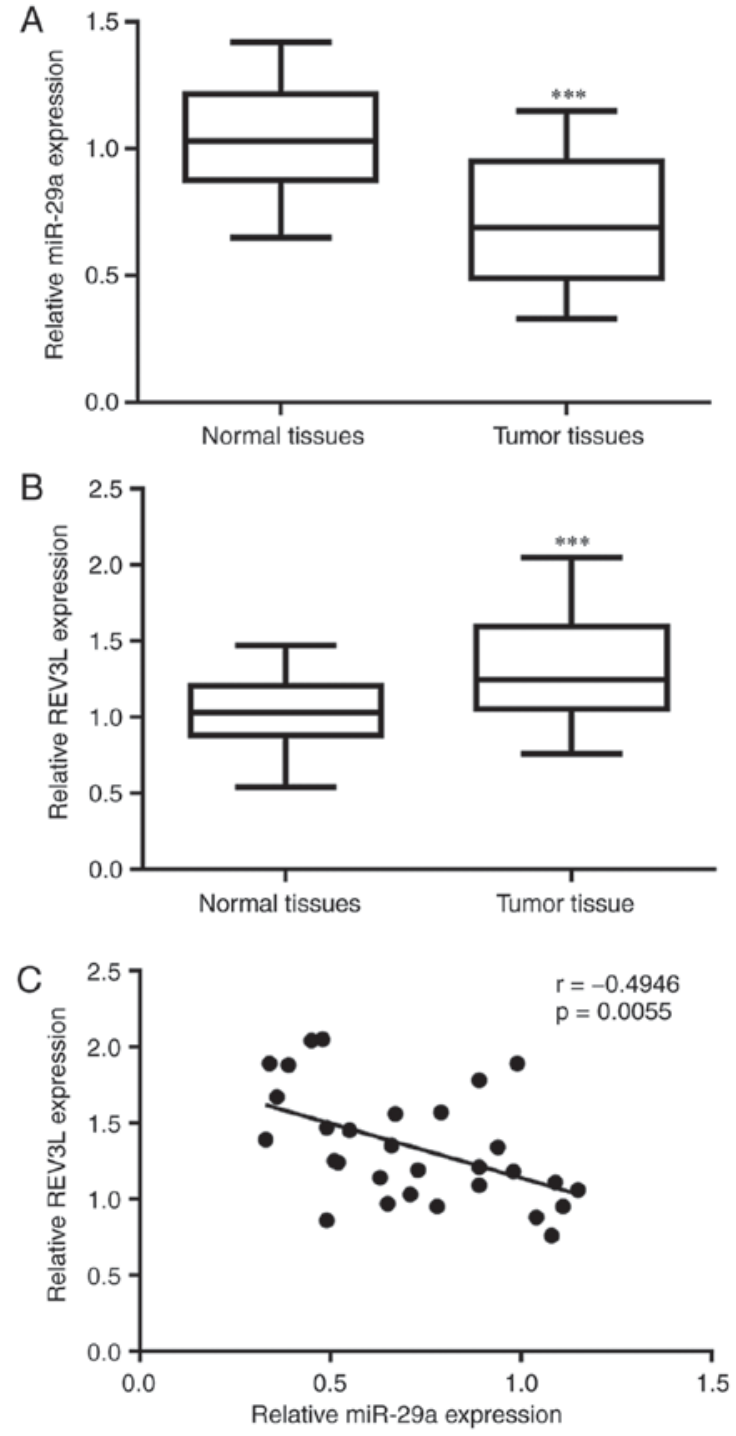

Figure 6. miR-29a expression is negatively correlated with REV3L mRNA levels in NSCLC tumor tissues. (A) miR-29a expression levels were significantly lower in tumoral tissues from patients with NSCLC compared with adjacent normal tissues. (B) REV3L mRNA levels were significantly elevated in tumoral tissues from patients with NSCLC compared with adjacent normal tissues. (C) A negative correlation was identified between miR-29a and REV3L mRNA expression levels in NSCLC tumor tissues, as determined by Pearson correlation analysis. ${ }^{* * *} \mathrm{P}<0.001$. miR, microRNA; NSCLC, non-small cell lung cancer; REV3L, REV3-like DNA-directed polymerase $\zeta$ catalytic subunit.

miR-29a downregulates REV3L expression in NSCLC cells. REV3L is involved in the DNA repair pathway and is an indicator of chemotherapy sensitivity in several cancer types (18). In A549 and H1650 cells, overexpression of miR-29a significantly decreased REV3L mRNA expression, compared with miR-NC-transfected cells (Fig. 3A). Western blot analysis further demonstrated a notable reduction of REV3L protein expression in miR-29a-transfected cells (Fig. 3B). In addition, higher REV3L mRNA and protein expression levels were detected in A549rCDDP cells, compared with A549 cells (Fig. 3C and D). A549rCDDP cells transfected with miR-29a mimics exhibited a significant reduction in REV3L mRNA and protein expression levels, compared with miR-NC-transfected cells (Fig. 3E and F). These results suggested a potential 
role for miR29a and REV3L in the development of cisplatin resistance.

REV3L was a direct target of miR-29a. TargetScan 7.1 was used to predict target sequences of miR-29a in the 3'UTR of REV3L (Fig. 4A). To confirm the direct regulatory relationship between miR-29a and REV3L, a dual luciferase assay was performed in 293 cells. Transfection of miR-29a mimics, but not miR-NC mimics, significantly reduced luciferase activity in cells transfected with REV3L 3'UTR-WT (Fig. 4B); no significant differences in luciferase activity were identified in cells co-transfected with REV3L 3'UTR-Mut. These data demonstrated that miR-29a may inhibit REV3L expression by binding to its 3'UTR.

miR-29a regulates cisplatin sensitivity of NSCLC cells through $R E V 3 L$. REV3L is the catalytic subunit of DNA polymerase $\zeta$, which is involved in translesional DNA synthesis (14). Variations in REV3L have previously been demonstrated to lead to altered cell cycle distribution and, therefore, an altered sensitivity to chemotherapy (18). REV3L siRNA1 and REV3L siRNA2 significantly decreased REV3L mRNA (Fig. 5A) and protein level (Fig. 5B) in A549 cells. Although treatment with a low concentration of cisplatin $(2 \mu \mathrm{g} / \mathrm{ml})$ alone (control siRNA + miR-NC mimics + cisplatin) did not affect the cell cycle compared with untreated control cells, a significant enrichment of cells in the G2/M phase was observed in REV3L-siRNA-transfected A549 cells treated with $2 \mu \mathrm{g} / \mathrm{ml}$ cisplatin (Fig. 5C and D). Similarly, miR-29a overexpression also induced G2/M arrest in A549 cells exposed to low-dose cisplatin (Fig. 5C and D). In addition, knockdown of REV3L expression or miR-29a overexpression significantly inhibited cell proliferation in the presence of cisplatin $(2 \mu \mathrm{g} / \mathrm{ml})$ compared with cells treated with cisplatin $(2 \mu \mathrm{g} / \mathrm{ml})$ only (Fig. 5E). These results further validated the potential role of miR-29a in the regulation of cisplatin sensitivity of A549 cells; this regulation may be achieved through the downregulation of REV3L expression and increased cisplatin-induced G2/M arrest.

miR-29a expression is inversely correlated with REV3L $m R N A$ expression in tumor tissues from patients with NSCLC. To investigate the function of miR-29a and REV3L in patients with NSCLC, RT-qPCR was used to detect miR-29a and REV3L mRNA expression levels in tumoral and adjacent normal tissues (Fig. 6). A significant decrease in miR-29a expression was observed in tumoral tissues compared with adjacent normal tissues (Fig. 6A), whereas REV3L mRNA expression was elevated in tumoral tissues compared with normal tissues (Fig. 6B). Notably, correlation analysis indicated that miR-29a expression was inversely correlated with REV3L mRNA expression in tumor tissues from patients with NSCLC (Fig. 6C). Statistical analysis of the associations between miR-29a expression, REV3L mRNA expression and clinicopathological factors reveled that low expression of miR-29a and high expression of REV3L were closely associated with advanced TNM stage (Table I). No significant associations were identified between miR-29a and REV3L expression with age, sex or metastatic status of the patients with NSCLC (Table I).

\section{Discussion}

Resistance towards cisplatin-based chemotherapy is a major obstacle for the treatment of patients with NSCLC (19). Although many proteins and miRNAs have been identified as drivers of chemoresistance (20-22), further investigation is required to elucidate the complicated underlying mechanisms. In the present study, miR-29a was revealed to potentially regulate the sensitivity of NSCLC cells to cisplatin treatment. miR-29a was also predicted and confirmed to directly regulate REV3L expression, and therefore antagonize cisplatin resistance in A549rCDDP cells.

Recently, a number of reports have demonstrated that dysregulation of miRNA networks leads to the development of drug resistance in a number of cancers $(23,24)$. In NSCLC, several miRNAs have been reported to contribute to the development of cisplatin resistance $(25,26)$. miR-29a was demonstrated to act as a tumor suppressor in NSCLC by regulating oncogene expression, such as LIM and $\mathrm{SH} 3$ protein 1, in several in vitro studies $(27,28)$. Consistent with these findings, the present study observed a decrease in miR-29a expression in NSCLC tumoral tissues. Downregulation of miR-29a has also been reported to be involved in the development of cisplatin resistance in ovarian cancer cells in vitro and in vivo (13). The present study revealed that altered miR-29a expression affected the sensitivity of NSCLC cells to cisplatin treatment. In A549 and H1650 cells, downregulation of miR-29a partially reversed cisplatin-induced cell growth arrest, whereas upregulation of miR-29a sensitized cells to cisplatin treatment. Furthermore, there was a notable decrease in miR-29a expression in cisplatin-resistant A549rCDDP cells compared to their A549 cell counterpart. Therefore, it was suggested that miR-29a may promote the sensitivity of NSCLC cells to cisplatin, and loss of miR-29a may be responsible for cisplatin resistance in NSCLC.

REV3L is the catalytic subunit of DNA polymerase $\zeta$, which is involved in translesional DNA synthesis (14). The role of REV3L in cancer progression is controversial. For example, low expression of REV3L was observed in colon carcinomas compared with normal tissues, which suggested a role as a tumor suppressor (29). By contrast, another study reported that REV3L depletion induced cell growth arrest in cancer cells of different origins, and identified REV3L as an oncogene (30). As for chemotherapy resistance, upregulation of REV3L was previously demonstrated to serve a crucial role in many cancer types, including NSCLC, through regulation of DNA repair $(16,31,32)$. In the present study, an elevated expression level of REV3L was observed in A549rCDDP cells. In addition, REV3L was predicted and confirmed to be directly regulated by miR-29a. Silencing of REV3L or overexpression of miR-29a inhibited cell growth and increased accumulation of cells in the G2/M phase in cells co-treated with cisplatin. This was consistent with a previous study that demonstrated that depletion of REV3L led to cumulative DNA damage, and resulted in inhibition of cell proliferation and G2/M arrest (30). Furthermore, results from the present study indicated that decreased miR-29a expression may contribute to the elevation of REV3L expression in A549rCDDP cells, and overexpression of miR-29a may reverse cisplatin resistance and induce growth arrest 
and apoptosis in A549rCDDP cells treated with cisplatin. Therefore, miR-29a/REV3L may promote the development of cisplatin resistance in NSCLC cells. The results of the present study provided further validation that REV3L depletion may amend cisplatin-based chemotherapy, and revealed that miR-29a may target REV3L to enhance cisplatin sensitivity of NSCLC cells.

In conclusion, the present study demonstrated that miR-29a positively regulates the sensitivity of NSCLC cells to cisplatin via direct suppression of REV3L expression. Downregulation of miR-29a led to cisplatin resistance in NSCLC cells and may be a promising prognostic tool and a target for the treatment of patients with NSCLC.

\section{Acknowledgements}

Not applicable.

\section{Funding}

This present work is supported by The Research Program on the Application of Public Welfare Technology in Zhejiang (grand no. 2016C33224), The Natural Science Foundation of Anhui (grant no. 1608085QH215) and The Medical Scientific Program of Shanghai Health and Family Planning Commission (grant no. 201540163).

\section{Availability of data and materials}

The datasets used and/or analyzed during the current study are available from the corresponding author on reasonable request.

\section{Authors' contributions}

$\mathrm{XC}, \mathrm{HZ}$ and WY performed the experiments and analyzed the data. YC analyzed the data. MC analyzed the data, collected the funding and prepared the manuscript.

\section{Ethics approval and consent to participate}

The present study was approved by the Ethics Committee of Zhejiang Cancer Hospital, and written informed consent was obtained from each patient prior to surgery and enrolment in the study.

\section{Patient consent for publication}

Patients consented to the publication of their clinical data.

\section{Competing interests}

The authors declare that they have no competing interests.

\section{References}

1. Torre LA, Bray F, Siegel RL, Ferlay J, Lortet-Tieulent J and Jemal A: Global cancer statistics, 2012. CA Cancer J Clin 65: 87-108, 2015

2. Riaz SP, Lüchtenborg M, Coupland VH, Spicer J, Peake MD and Møller H: Trends in incidence of small cell lung cancer and all lung cancer. Lung Cancer 75: 280-284, 2012.
3. Toschi L, Cappuzzo F and Janne PA: Evolution and future perspectives in the treatment of locally advanced non-small cell lung cancer. Ann Oncol 18 (Suppl 9): ix150-ix155, 2007.

4. Gu L, Deng JZ, Roy S and Hammond PT: A combination RNAi-chemotherapy layer-by-layer nanoparticle for systemic targeting of KRAS/P53 with cisplatin to treat non-small cell lung cancer. Clin Cancer Res 23: 7312-7323, 2017.

5. Barr MP, Gray SG, Hoffmann AC, Hilger RA, Thomale J, O'Flaherty JD, Fennell DA, Richard D, O'Leary JJ and O'Byrne KJ: Generation and characterisation of cisplatinresistant non-small cell lung cancer cell lines displaying a stem-like signature. PLoS One 8: e54193, 2013.

6. Siegel R, Ma J, Zou Z and Jemal A: Cancer statistics, 2014. CA Cancer J Clin 64: 9-29, 2014.

7. Bartel DP: MicroRNAs: Genomics, biogenesis, mechanism, and function. Cell 116: 281-297, 2004.

8. Lin S and Gregory RI: MicroRNA biogenesis pathways in cancer. Nat Rev Cancer 15: 321-333, 2015.

9. Pan JY, Sun CC, Bi ZY, Chen ZL, Li SJ, Li QQ, Wang YX, Bi YY and Li DJ: miR-206/133b Cluster: A Weapon against lung cancer? Mol Ther Nucleic Acids 8: 442-449, 2017.

10. Ni J, Bucci J, Chang L, Malouf D, Graham P and Li Y: Targeting MicroRNAs in prostate cancer radiotherapy. Theranostics 7 : 3243-3259, 2017.

11. Ayers D and Vandesompele J: Influence of microRNAs and long non-coding RNAs in cancer chemoresistance. Genes 8: pii: E95, 2017.

12. Pal MK, Jaiswar SP, Dwivedi VN, Tripathi AK, Dwivedi A and Sankhwar P: MicroRNA: A new and promising potential biomarker for diagnosis and prognosis of ovarian cancer. Cancer Biol Med 12: 328-341, 2015.

13. Yu PN, Yan MD, Lai HC, Huang RL, Chou YC, Lin WC, Yeh LT and Lin YW: Downregulation of miR-29 contributes to cisplatin resistance of ovarian cancer cells. Int J Cancer 134: 542-551, 2014.

14. Lawrence CW and Hinkle DC: DNA polymerase zeta and the control of DNA damage induced mutagenesis in eukaryotes. Cancer Surv 28: 21-31, 1996.

15. Zhang S, Chen H, Zhao X, Cao J, Tong J, Lu J, Wu W, Shen H, Wei Q and Lu D: REV3L 3'UTR $460 \mathrm{~T}>\mathrm{C}$ polymorphism in microRNA target sites contributes to lung cancer susceptibility. Oncogene 32: 242-250, 2013.

16. Wu F, Lin X, Okuda T and Howell SB: DNA polymerase zeta regulates cisplatin cytotoxicity, mutagenicity and the rate of development of cisplatin resistance. Cancer Res 64: 8029-8035, 2004.

17. Livak KJ and Schmittgen TD: Analysis of relative gene expression data using real-time quantitative PCR and the 2- $\Delta \Delta \mathrm{CT}$ method. Methods 25: 402-408, 2001

18. Yang L, Shi T, Liu F, Ren C, Wang Z, Li Y, Tu X, Yang G and Cheng X: REV3L, a promising target in regulating the chemosensitivity of cervical cancer cells. PLoS One 10: e0120334, 2015.

19. Hu Y, Hong Y, Xu Y, Liu P, Guo DH and Chen Y: Inhibition of the JAK/STAT pathway with ruxolitinib overcomes cisplatin resistance in non-small-cell lung cancer NSCLC. Apoptosis 19: 1627-1636, 2014

20. He J, Yu JJ, Xu Q, Wang L, Zheng JZ, Liu LZ and Jiang BH: Downregulation of ATG14 by EGR1-MIR152 sensitizes ovarian cancer cells to cisplatin-induced apoptosis by inhibiting cyto-protective autophagy. Autophagy 11: 373-384, 2015.

21. Li J, Wang Y, Song Y, Fu Z and Yu W: miR-27a regulates cisplatin resistance and metastasis by targeting RKIP in human lung adenocarcinoma cells. Mol Cancer 13: 193, 2014.

22. Wu DW, Wu TC, Wu JY, Cheng YW, Chen YC, Lee MC, Chen $\mathrm{CY}$ and Lee $\mathrm{H}$ : Phosphorylation of paxillin confers cisplatin resistance in non-small cell lung cancer via activating ERK-mediated Bcl-2 expression. Oncogene 33: 4385-4395, 2014.

23. Dehghanzadeh R, Jadidi-Niaragh F, Gharibi T and Yousefi M: MicroRNA-induced drug resistance in gastric cancer. Biomed Pharmacother 74: 191-199, 2015.

24. Zou J, Yin F, Wang Q, Zhang W and Li L: Analysis of microarray-identified genes and microRNAs associated with drug resistance in ovarian cancer. Int J Clin Exp Pathol 8: 6847-6858, 2015.

25. Dong Z, Zhong Z, Yang L, Wang S and Gong Z: MicroRNA-31 inhibits cisplatin-induced apoptosis in non-small cell lung cancer cells by regulating the drug transporter ABCB9. Cancer Lett 343: 249-257, 2014. 
26. Ma Y, Li X, Cheng S, Wei W and Li Y: MicroRNA-106a confers cisplatin resistance in non-small cell lung cancer A549 cells by targeting adenosine triphosphatase-binding cassette A1. Mol Med Rep 11: 625-632, 2015.

27. Muniyappa MK, Dowling P, Henry M, Meleady P, Doolan P, Gammell P, Clynes M and Barron N: MiRNA-29a regulates the expression of numerous proteins and reduces the invasiveness and proliferation of human carcinoma cell lines. Eur J Cancer 45 3104-3118, 2009.

28. Hu Z, Cui Y, Zhou Y, Zhou K, Qiao X, Li C and Wang S: MicroRNA-29a plays a suppressive role in non-small cell lung cancer cells via targeting LASP1. Onco Targets Ther 9: 6999-7009, 2016.

29. Brondello JM, Pillaire MJ, Rodriguez C, Gourraud PA, Selves J, Cazaux $\mathrm{C}$ and Piette $\mathrm{J}$ : Novel evidences for a tumor suppressor role of Rev3, the catalytic subunit of Pol zeta. Oncogene 27: 6093-6101, 2008.
30. Knobel PA, Kotov IN, Felley-Bosco E, Stahel RA and Marti TM: Inhibition of REV3 expression induces persistent DNA damage and growth arrest in cancer cells. Neoplasia 13: 961-970, 2011

31. Wang H, Zhang SY, Wang S, Lu J, Wu W, Weng L, Chen D, Zhang Y, Lu Z, Yang J, et al: REV3L confers chemoresistance to cisplatin in human gliomas: the potential of its RNAi for synergistic therapy. Neuro Oncol 11: 790-802, 2009.

32. Wang W, Sheng W, Yu C, Cao J, Zhou J, Wu J, Zhang H and Zhang S: REV3L modulates cisplatin sensitivity of non-small cell lung cancer H1299 cells. Oncol Rep 34: 1460-1468, 2015.

cc (i) $\Theta$ This work is licensed under a Creative Commons Attribution-NonCommercial-NoDerivatives 4.0 International (CC BY-NC-ND 4.0) License. 